\title{
Biophysically Inspired Development of a Sand-Swimming Robot
}

\author{
Ryan D. Maladen*, Yang Ding ${ }^{\dagger}$, Paul B. Umbanhowar ${ }^{\ddagger}$, Adam Kamor ${ }^{\dagger}$ and Daniel I. Goldman*† \\ *Bioengineering Program, ${ }^{\dagger}$ School of Physics, Georgia Institute of Technology, Atlanta, Georgia 30332-0250 \\ ${ }_{\ddagger}^{\ddagger}$ Department of Mechanical Engineering, Northwestern University, Evanston, IL 60208 \\ email: daniel.goldman@physics.gatech.edu
}

\begin{abstract}
Previous study of a sand-swimming lizard, the sandfish, Scincus scincus, revealed that the animal swims within granular media at speeds up to 0.4 body-lengths/cycle using body undulation (approximately a single period sinusoidal traveling wave) without limb use [1]. Inspired by this biological experiment and challenged by the absence of robotic devices with comparable subterranean locomotor abilities, we developed a numerical simulation of a robot swimming in a granular medium (modeled using a multi-particle discrete element method simulation) to guide the design of a physical sand-swimming device built with off-the-shelf servo motors. Both in simulation and experiment the robot swims limblessly subsurface and, like the animal, increases its speed by increasing its oscillation frequency. It was able to achieve speeds of up to 0.3 body-lengths/cycle. The performance of the robot measured in terms of its wave efficiency, the ratio of its forward speed to wave speed, was $0.34 \pm 0.02$, within $8 \%$ of the simulation prediction. Our work provides a validated simulation tool and a functional initial design for the development of robots that can move within yielding terrestrial substrates.
\end{abstract}

\section{INTRODUCTION}

There is a need for robots that can move within complex material like sand, rubble, and loose debris. For example such robots could help locate hazardous chemical leaks [2], function as self propelled inspection devices [3], and search for victims in disaster sites $[4,5,6]$. Limbless robots that use their bodies to move appear better suited to navigate complex terrains than traditional wheeled $[7,8,9,10]$ and legged $[11,12,13,14$, $15,16,17]$ robots which are often impeded by the size or shape of their appendages which can result in entrapment or failure. Previous terrestrial limbless robots utilized serpentine locomotion to move on the surface of media. Of these, most were tested on rigid surfaces $[18,19,20,21,22]$ with only a few developed for and tested in unstructured environments [23, 24, 25].

Advances in creating high performing flying and swimming devices $[22,26,27]$ in aerial and aquatic domains and wheeled/tracked vehicles on relatively structured terrestrial terrain have occurred mainly because the respective fields of aerodynamics, fluid dynamics, and terramechanics [28, 26] provide accurate models of locomotor-media interaction which are used in turn to design improved wings, fins, wheels, and legs. However, a major hurdle arises when one attempts to design robots to move on and within complex flowing particulate environments (e.g. sand, soil, and leaf-litter) that can display both solid and fluid-like behavior in response to stress. In such materials, comparable and comprehensive val- idated analytic continuum theories at the level of the NavierStokes equations [29] for fluids do not exist. However, it is possible to understand the interaction between the locomotor and the media by using numerical and physical modeling approaches [30, 31, 32].

In the absence of theory, the biological world is a fruitful source of principles of movement that can be incorporated into the design of robots that navigate within complex substrates. Many desert organisms like scorpions, snakes, and lizards burrow and swim effectively in sand [33, 34, 35, 36, 37] to escape heat and predators, and hunt for prey [38, 39]. It has been hypothesized that many of these animals have evolved morphological adaptations like marked body elongation and limb reduction to deal with deformable terrain [40, 41]. Our recent high speed $x$-ray imaging study investigating the subsurface locomotion of the sandfish Scincus scincus, a small $(\sim 10 \mathrm{~cm}$ long snout - tail tip) lizard that inhabits the Saharan desert [1] (Fig. 1), reveals that once within the media the animal no longer uses limbs for propulsion but "swims" forward by propagating a sinusoidal traveling wave posteriorly from head to tail.

Motivated by the subsurface locomotion of the sandfish, the present work utilizes a numerical simulation of a sandfish inspired undulator as a design tool to build an appendageless sand-swimming device. The robot is driven by a simple open loop controller which, like the animal kinematics, varies the joint position trajectories to create a sinusoidal wave that travels posteriorly along the device. The robot swims within a model laboratory granular medium of plastic particles and displays locomotion features similar to the organism and predicted by the numerical robot simulation.

\section{PREVIOUS WORK}

\section{A. Biological Experiment}

The biological experiments presented in [1] model the subsurface undulatory motion of the sandfish with a posteriorly traveling single-period sinusoidal wave

$$
y=A \sin \frac{2 \pi}{\lambda}\left(x+v_{w} t\right)
$$

with $x$ the position along the sandfish, $y$ the body displacement from the midline of the animal, $A$ the amplitude, $\lambda$ the wavelength and $v_{w}=f \lambda$ the wave speed where $f$ is the wave frequency. The spatial characteristics, $A$ and $\lambda$, did 


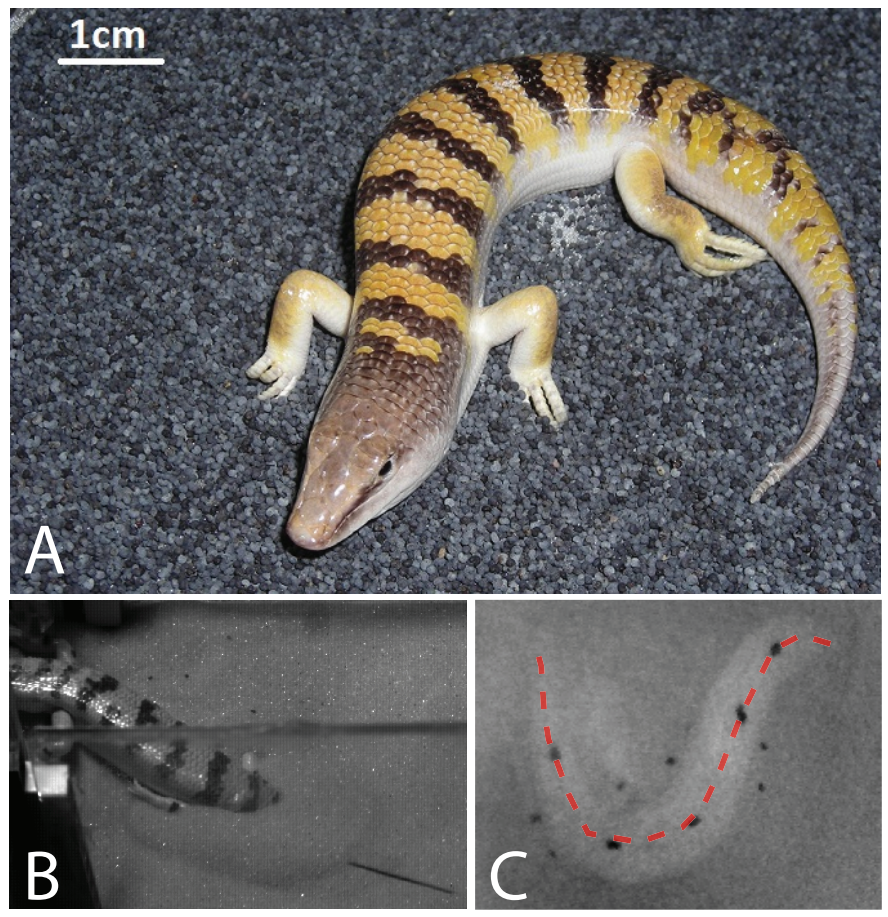

Fig. 1. (A) The sandfish Scincus scincus, a sand-swimming lizard that inhabits the Saharan desert, (B) burying into granular media $(0.3 \mathrm{~mm}$ spherical glass beads), and (C) swimming subsurface where the x-ray image shows the body (light area) and opaque markers fixed to limbs and midline. Red dashed line indicates tracked midline.

not vary significantly with media preparation and their ratio was approximately 0.2 implying that the animal increased its forward velocity by increasing its oscillation frequency.

A measure of undulatory performance is the wave efficiency, $\eta$, the ratio between the forward speed of the swimmer, $v_{x}$, and the velocity of the wave traveling down its body, $v_{w}$, or equivalently the slope of the velocity-frequency relationship for velocity measured in wavelengths per second. Typical wave efficiencies of undulatory organisms moving in fluids at low Reynolds number (such as nematodes in water) are 0.25 [42, 43, 44], whereas $\eta \approx 0.8-0.9$ for organisms undulating (creeping) along solid - air interfaces [45, 46, 47]. Locomotion with $\eta=1$ is equivalent to movement within a rigid tube. For the sandfish swimming in glass beads, $\eta \approx 0.5$ independent of particle size and media preparation (i.e. packing density).

\section{B. Resistive Force Theory for Granular Media}

An empirical resistive force theory (RFT) was developed to predict wave efficiency $\eta$ for undulatory subsurface granular locomotion [1]. The RFT, inspired by theory used to predict swimming speeds of microorganisms in fluids [42], partitions the body of the organism into infinitesimal segments each of which generates thrust and experiences drag when moving through the medium. These segmental forces are integrated over the entire body, and, by setting the net forward force to zero (assuming a constant average velocity), $\eta$ is solved for numerically.
Unlike fluids, in granular media no validated theory exists in the regime relevant to sand-swimming to estimate the force on individual segments moving through the medium. Previously, Maladen et al. [1] obtained these forces empirically by dragging a rod (representative segment) through the media the animal was tested in. With these forces as input and by propagating a sinusoidal traveling wave along the body, the RFT shows that translational motion within granular media without limb use is possible. Also, the RFT accurately predicts that the sandfish swims with $\eta \approx 0.5$ within a granular media of $0.3 \mathrm{~mm}$ glass particles (comparable in size and density to desert sand [48]).

While the RFT qualitatively describes some features of sand-swimming, it is based on several assumptions: e.g. the measured drag force on a rod is representative of the average force on a segment of the sandfish, the forces generated by a segment are localized, and the center of mass of the animal does not oscillate laterally. Since the assumptions of the RFT have not been rigourously tested and applying the RFT to different treatments (particle friction, particle size, body design, etc.) require force laws to be measured for each condition, we instead use numerical simulation techniques as a general robotic design tool.

A numerical simulation approach, once validated against experiment, can provide an understanding of body generated drag and thrust forces from the particle perspective and can be used to generate empirical drag laws for input into the RFT. Our numerical simulation is a flexible design tool that accurately predicts robot performance and allows easy variation of physical and design parameters such as particle-particle friction and number of segments.

\section{Numerical Simulation OF SAND-SWimming ROBOT}

\section{A. Development and Validation}

To design a sand-swimming robot, we developed a numerical simulation of a laboratory scale device with a finite number of discrete, rigid segments to test if it could swim within granular medium. The simulation couples a numerical model of the robot to a model of the granular medium. We model the granular material using a multi-particle discrete element method (DEM) simulation [30]. To compute the robot-particle and particle-particle interaction forces we calculate the normal force [49], $F_{n}$, and the Coulombic tangential force, $F_{s}$, acting at each contact with

$$
\begin{aligned}
F_{n} & =k \delta^{3 / 2}-G_{n} v_{n} \delta^{1 / 2} \\
F_{s} & =\mu F_{n},
\end{aligned}
$$

where $\delta$ is the virtual overlap between particles or between particle and robot segment, $v_{n}$ is the normal component of relative velocity, and $k=2 \times 10^{5} \mathrm{~kg} \mathrm{~s}^{-2} \mathrm{~m}^{-1 / 2}$ and $G_{n}=$ $5 \mathrm{~kg} \mathrm{~s}^{-1} \mathrm{~m}^{-1 / 2}$ are the hardness and viscoelastic constant. $\mu$ quantifies the particle-particle $\left(\mu_{p p}=0.08\right)$ or body-particle $\left(\mu_{b p}=0.27\right)$ friction coefficient depending on which elements are in contact. $\mu_{b p}$ was measured between the robot skin 
and plastic particles used in the physical experiments. To reduce the required torque in the physical experiments and to decrease the computational time, we used a granular medium composed of $4.7 \times 10^{5}$ spherical plastic particles with diameter $6 \mathrm{~mm}$ and density $1.03 \pm 0.04 \mathrm{~g} / \mathrm{cm}^{3}$ in experiment, and $3.2 \times 10^{5}$ bi-disperse (50:50) sphere mixture $(5.81,5.93 \mathrm{~mm})$ and density $1.06 \mathrm{~g} / \mathrm{cm}^{3}$ in simulation. The 35 particle deep bed of particles in experiment and 24 particle deep bed of particles in simulation were held in a container with horizontal cross section of $188 \times 62$ particle diameters. To validate the simulated medium and obtain the values of $\mu_{p p}, k$, and $G_{n}$ given above, we dropped an aluminum ball (diameter $6.35 \mathrm{~cm}$ and mass $385 \mathrm{~g}$ ) into the plastic particles with varying impact velocity $(0.5-3 \mathrm{~m} / \mathrm{s})$ in both experiment and simulation and set grain interaction parameters to best match the measured and simulated penetration force during the impact collision as a function of time (Fig. 2). With parameters determined from impact at $v=1.4 \mathrm{~m} / \mathrm{s}$, the force profile fit well at other impact velocities. In additional experiments, we directly measured $\mu_{p p}$ and the coefficient of restitution (determined by $G_{n}$ with fixed $k$ ) for the plastic particles and found them to be within $5 \%$ and $10 \%$ of the fitted values respectively. For simplicity we used the same normal force parameters for both particle-particle and body-particle interactions.

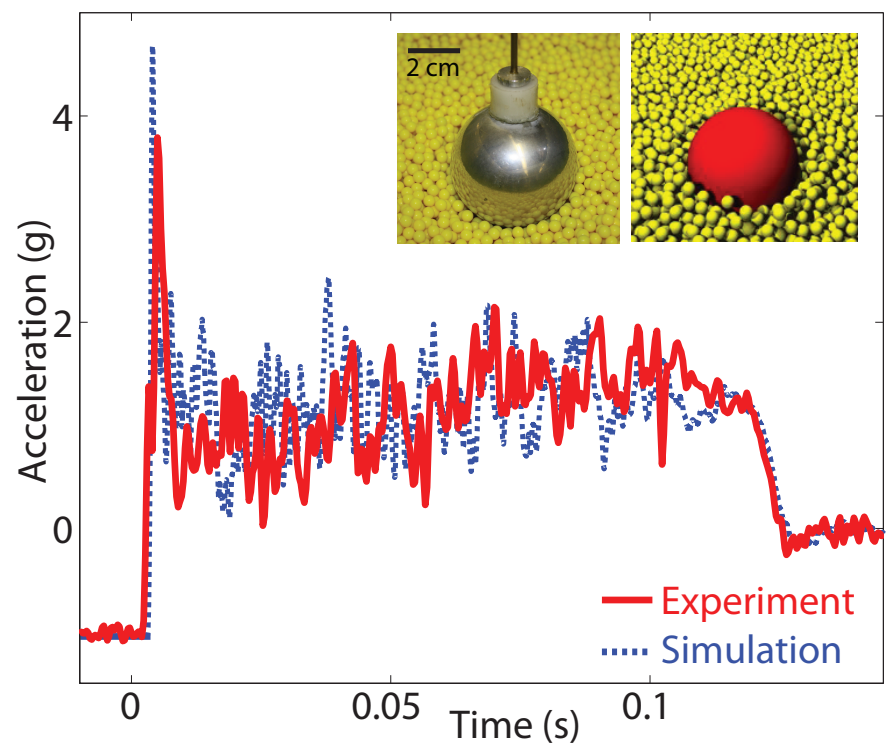

Fig. 2. Validation of the multi-particle discrete element method (DEM) simulation of the granular medium using measured acceleration of a sphere during vertical impact after free-fall. Acceleration vs. time in simulation (blue dashed trace) and experiment (red solid trace) agree well. The impact velocity for this representative run is $1.4 \mathrm{~m} / \mathrm{s}$. Acceleration is in units of $g$, the acceleration due to gravity. (Left inset) Aluminium ball instrumented with accelerometer resting on $6 \mathrm{~mm}$ plastic particles. (Right inset) Ball and particles in simulation.

To model the sand-swimming device we used the commercial multi-body simulator software package Working Model (WM) 2D (Design Simulation Technologies). Modeling the device in a $2 \mathrm{D}$ simulation environment is sufficient to capture the dynamics since the sand-swimming robot moves roughly in a horizontal plane at fixed depth in this study, and there was excellent agreement between experiment and simulation in preliminary studies. The simulated robot was sized for easy testing of the corresponding physical device in the laboratory. Since the sandfish does not use its limbs to move subsurface and RFT had shown that body undulation was sufficient for propulsion [1], the simulated robot did not include limbs. No tapering along the device was considered. The simulated robot consisted of 49 cuboidal segments interconnected and actuated by virtual motors (vertical cylinders) of the same height (Fig. 3). Depending on the number of segments $(N)$ employed, every $48 / N$ motor was driven with an open loop signal to generate a sinusoidal wave traveling posteriorly from head to tail while the remaining motors were immobilized to form a straight segment of length $48 / N \mathrm{~cm}$. To approximate a sinusoidal traveling wave, the angle between adjacent segments is modulated using

$$
\beta(i, t)=\beta_{0} \xi \sin (2 \pi \xi i / N-2 \pi f t),
$$

with $\beta(i, t)$ the motor angle of the $i^{t h}$ motor at time $t, \beta_{0}$ the angular amplitude which determines $A / \lambda, \xi$ the number of wavelengths along the body (period), and $N$ the number of motors.

WM integrates the equations of motion of the coupled links and the DEM calculates the resultant forces from both the particle-particle and body-particle interactions. For each time step, the net force from particles on each segment is passed to WM, and velocity and position information transferred back to DEM. Roll and pitch were not modeled.

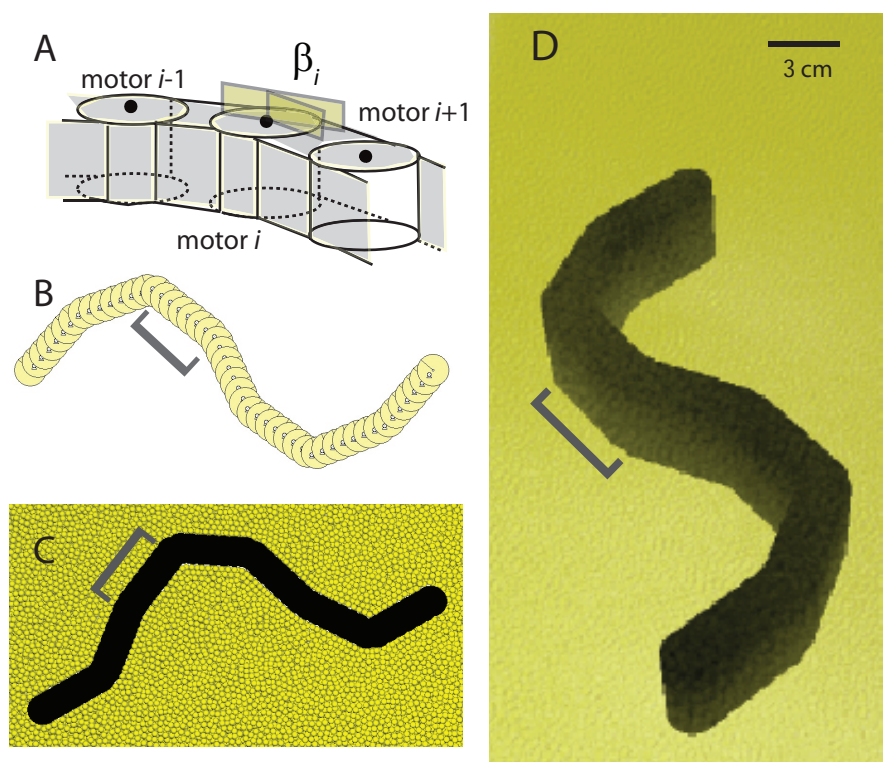

Fig. 3. Simulation of a sand-swimming robot. (A,B) Side and top view of the robot modeled with 49 inter-connected motor segments and one head segment. The angle between adjacent motors $\left(\beta_{i}\right)$ is modulated using Eqn. 3 to reproduce the sandfish's sinusoidal traveling wave kinematics. (C) Top view of the device submerged in $6 \mathrm{~mm}$ particles with particles above the robot rendered transparent. (D) Rendering of the simulated robot for the same parameters used in robot experiment (see Fig. 4). The brackets ( [ ) indicate a single robot segment. 
Using Eqn. 3 the simulated robot with 7 total segments moved forward within $6 \mathrm{~mm}$ plastic particles and increased its forward speed linearly with oscillation frequency (Fig. 7). The wave efficiency was $\eta=0.36 \pm 0.02$, less than that of the sandfish lizard. Motivated by these results we built a physical instantiation of the scaled model.

\section{SAND SWIMMING RobOT}

\section{A. Design and Control}

The basic mechanical design of our device was adapted from previously developed snake robots [25] which consisted of repeated modules (motors) each with a single joint that permit angular excursions in a plane and connected via identical links. In our design, each module consists of a servomotor attached to an aluminium bracket and is connected to adjacent motors via the brackets. The wire bundle that routes power and control signals to each motor was run atop each module over the length of the device and strain relieved at the last (tail) segment. For convenience and to maintain a reasonable size, our device employed 6 standard size servomotors and a dummy segment (the head) with the same weight and form factor as the motor segments for a total of 7 segments (Fig. 4A).

The simulation found that the peak torque required to swim subsurface at a depth of $4 \mathrm{~cm}$ was $0.7 \mathrm{Nm}$. To verify this finding we dragged an object with the same form factor as a motor through the $6 \mathrm{~mm}$ plastic medium at $0.25 \mathrm{~m} / \mathrm{s}$. The measured force at a depth of $4 \mathrm{~cm}$ was $3.2 \mathrm{~N}$. Since the maximum torque occurs at the middle motor $(0.23 \mathrm{~m}$ to either end) we estimated the maximum possible total force along an effective segment extending from the middle servo to either the tail or the head (length $0.23 \mathrm{~m}$ ) to be $18 \mathrm{~N}$ with a corresponding maximum torque of $2.0 \mathrm{Nm}$. We selected a servomotor that exceeded both torque estimates, see Table I.

Servomotors are powered in parallel from a $7.4 \mathrm{~V}, 30 \mathrm{~A}$ supply. The pulse width based control signal for each motor is generated in LabVIEW using Eqn. 3 as a multiplexed signal, output from a PCI-card (NI-6230), and connected to the clock input of a decade counter (CD4017BC) which functions as a demultiplexer and distributes a control pulse to each motor every $20 \mathrm{~ms}$.

Since the robot operates in a granular medium it is critical to encase it in a material that prevents particles from getting between the motor segments but allows the device to easily undulate. After testing several materials we found that a 2layer encasement consisting of an outer Lycra spandex sleeve with a single seam (located at the top of the device) enclosing an inner thin latex sleeve that fit tautly around the motors was satisfactory (Fig. 4).

\section{B. Experimental Methods}

We tested the robot in a container of the same dimensions as used in the robot simulations and filled with $6 \mathrm{~mm}$ plastic particles prepared in an as-poured state [50]. Overhead video (100 fps) was collected for each condition tested. To facilitate subsurface tracking the first and last module were fixed with
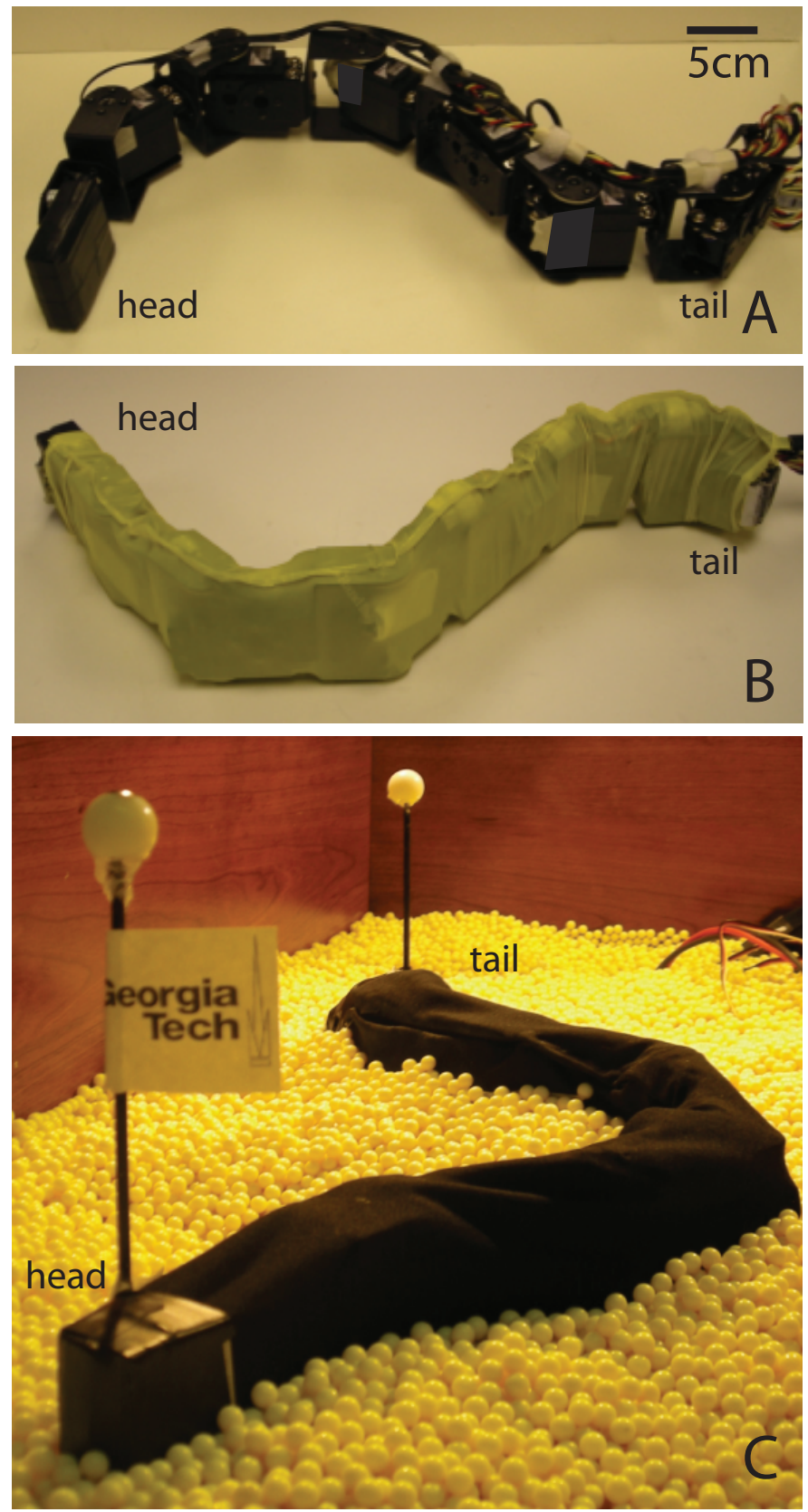

Fig. 4. Prototype of the sand-swimming robot. (A) basic construction (servomotors and aluminium brackets with power wires running along the top of the device). The robot has a double layer skin: (B) tight fitting thin latex inner layer, and (C) Lycra spandex outer layer. Balls on narrow masts on the head and tail segments allow subsurface motion tracking.

a mast with a visible marker. The wire bundle was run up the mast on the last segment and tethered above the container. The kinematics of the subsurface motion of the robot were also obtained using $\mathrm{x}$-ray imaging for a representative condition ( $f=0.25 \mathrm{~Hz}, A / \lambda=0.2$ ), see Fig. 5A-C. For each test the top of the robot was submerged $4 \mathrm{~cm}$ below the surface and the surface leveled. Due to the servomotor angular velocity limits the maximum oscillation frequency was $1 \mathrm{~Hz}$. For each frequency, $1-2$ cycles of motion were collected. 


\begin{tabular}{|l|l|}
\hline Dimensions & $0.48 \times 0.028 \times 0.054 \mathrm{~m}^{3}$ \\
Mass & $0.83 \mathrm{~kg}$ \\
Motor & HSR-5980SG \\
Motor Torque & $2.94 \mathrm{~N} \mathrm{~m}$ \\
Number of Motors & 6 \\
Total Segments & 7 \\
\hline
\end{tabular}

TABLE I

PHYSICAL ROBOT CHARACTERISTICS
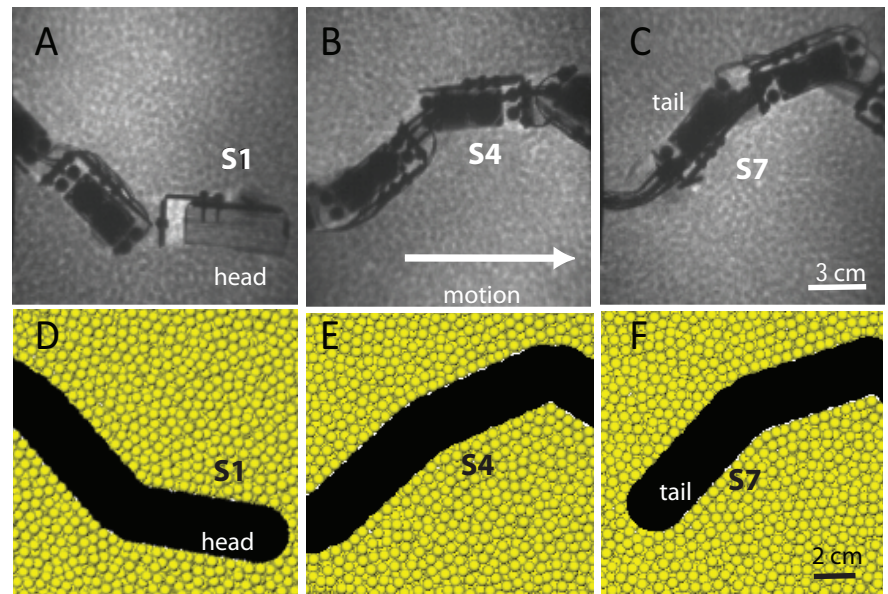

Fig. 5. Subsurface swimming in experiment and simulation. (A-C) Sequential $\mathrm{x}$-ray images of the robot swimming in $6 \mathrm{~mm}$ particles, and (D-F) robot swimming in simulation. Segments from head to tail are denoted as S1 to S7.

\section{Robot Performance}

To calibrate the device we placed it on a rigid surface and used video to track the position of the segments from which we determined the mapping between the maximum relative segment angle $\beta_{0}$ (Eqn. 3) and $A / \lambda$. Within the granular material, the forward velocity of the device monotonically increased with increasing oscillation frequency (Fig. 7) for $A / \lambda=0.2$ and a single period wave. The slope of this relationship $(\eta)$ was $0.34 \pm 0.02$. For the same parameters the simulation predicted $\eta=0.36 \pm 0.02$.

\section{DiscusSION}

Like the sandfish, the robot swims within granular media by propagating a traveling sinusoidal wave posteriorly from head to tail without limb use. The physical device demonstrates that subsurface locomotion in granular media using a relatively low degree of freedom device and a open loop control scheme is possible. However, the robot does not move forward as fast (normalized by body-length) or with the same wave efficiency as the animal.

In the biological experiments, $\eta$ for a range of granular material preparations and bead size was approximately 0.5. The robot in both experiment and simulation performed below this value. We hypothesized that the number of segments (for a fixed length device) affected both $\eta$ and the forward speed of the device. Increasing the number of segments in the robot simulation caused the device to move forward faster and with greater wave efficiency until $N \sim 15$ where $\eta$
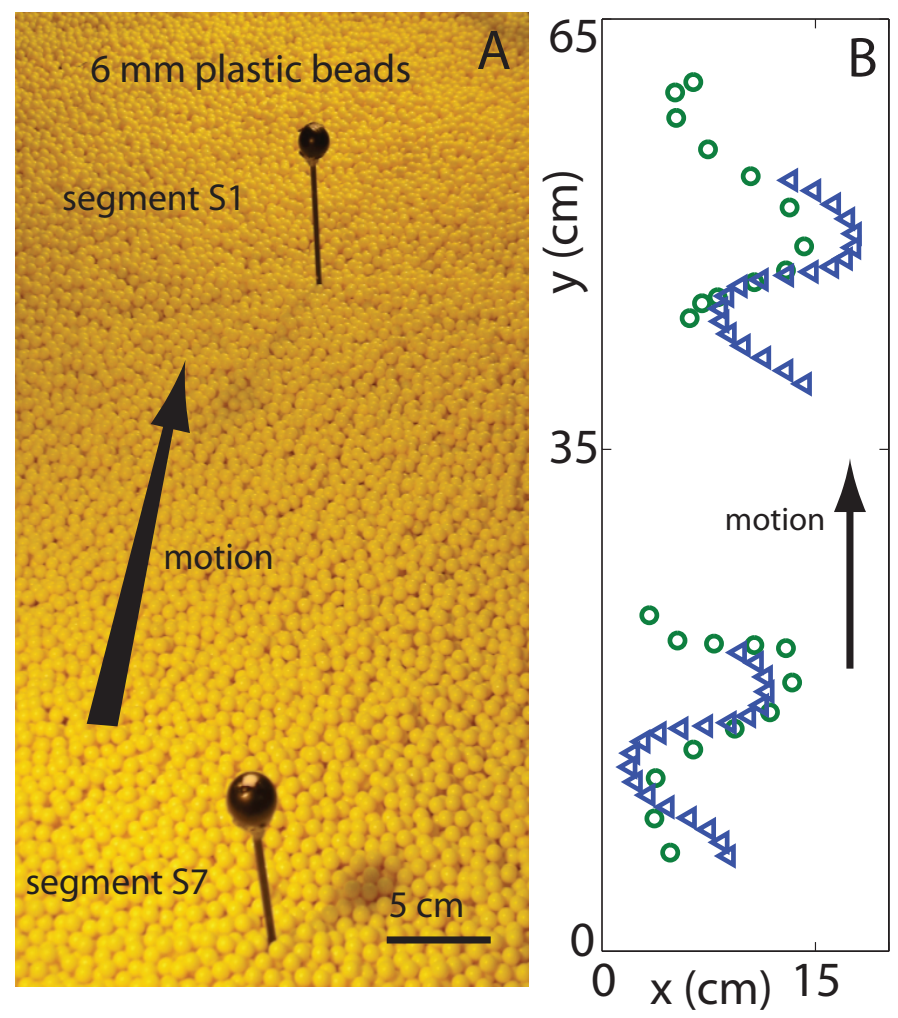

Fig. 6. Subsurface swimming in experiment and simulation. (A) Robot submerged in a container filled with $6 \mathrm{~mm}$ plastic particles. Masts with spherical markers are attached to the first and last module. (B) Kinematics of the first and last segment of the robot in experiment (green circles) and simulation (blue triangles).

plateaued (Fig. 8). Interestingly, the maximum $\eta \approx 0.5$ is the same as measured in the animal experiment. We utilized our previously developed RFT to predict the performance of the sand-swimming device with parameters set to match those for the plastic particles used in the robot experiment. We estimated $\eta=0.56$ for a smooth profiled undulator which corresponds to the numerical robot simulation prediction for $N>15$ (gray band, Fig. 8).

Increasing $N$ allowed the device to better match a sinusoidal wave and increased $\eta$ This suggests that deviation from the smooth form of a traveling sinusoidal wave reduces performance. A seven segment robot operates below the minimum $N$ required to achieve maximum $\eta$. As a design criterion, $N$ is important when the length of the device is fixed as increasing the number of motors beyond the critical $N$ requires motors with smaller dimensions but capable of producing the same torque.

We used the numeric robot simulation to measure the time varying torque required to move within the medium. As expected, the torque was approximately sinusoidal for all motors and the torque amplitude generated by the central motors ( 3 and 4) was larger than the torque from the motors nearest the ends, see Fig. 9. As noted earlier, the maximum torque in the simulation of $0.7 \mathrm{Nm}$ was well below the maximum of the motors used in experiment (see Table 1). 


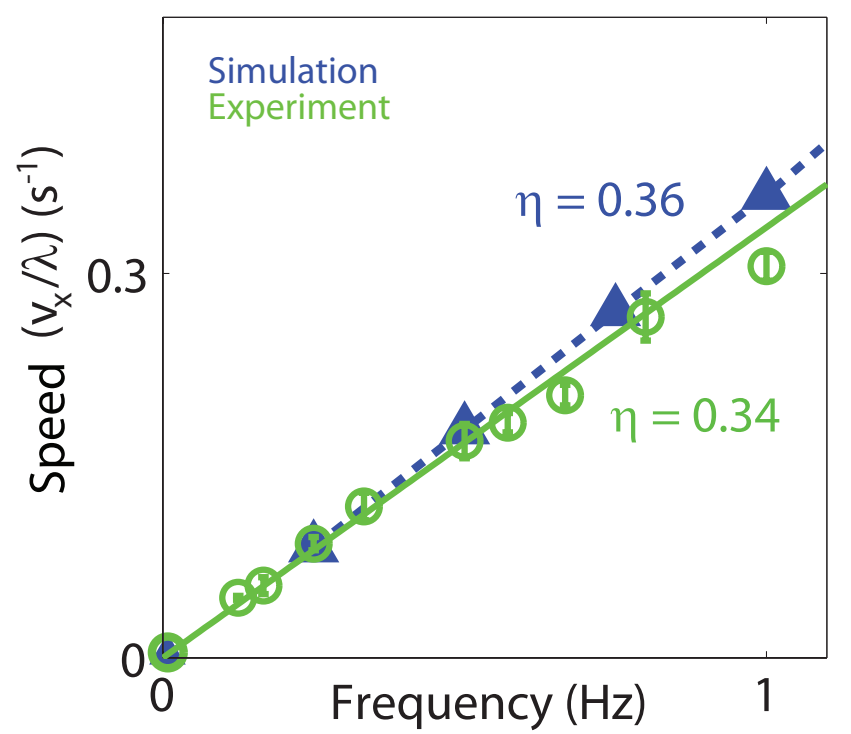

Fig. 7. Forward velocity vs. oscillation frequency for the robot in experiment (green circles) and simulation (blue triangles) $(A / \lambda=0.2)$. The slope of the dashed (simulation) and solid (experiment) fit lines gives the wave efficiency $\eta$.

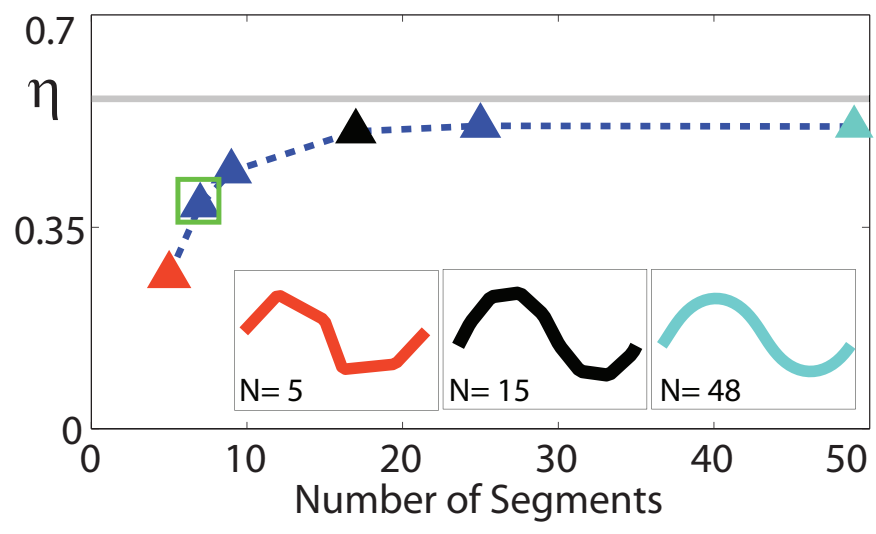

Fig. 8. Wave efficiency increases with number of segments for a fixed length robot in simulation (blue dashed curve $)(f=1 \mathrm{~Hz}$ and $A / \lambda=0.2)$. The red, black, and cyan triangles correspond to 5, 15, and 48 segment robots respectively. The green square corresponds to the seven segment physical robot, and the grey line indicates $\eta$ predicted by the RFT solved for a continuous body profile (see text for details).

Also, the fluctuations in torque at frequencies higher than the oscillation frequency of the robot were small in comparison to the torque amplitude.

\section{FUTURE WORK}

A sand swimming robot combined with a proven simulation tool opens many avenues for further research. Of immediate interest is testing the RFT prediction that an optimal spatial form (ratio of amplitude to wavelength) maximizes forward speed of an undulatory sand swimmer [1]. The effect of the predicted optimal kinematics can also be evaluated by measuring the mechanical cost of transport. In conjunction with the numerical simulation the robot can test the effect of motion profiles (wave shapes) on performance. Since the

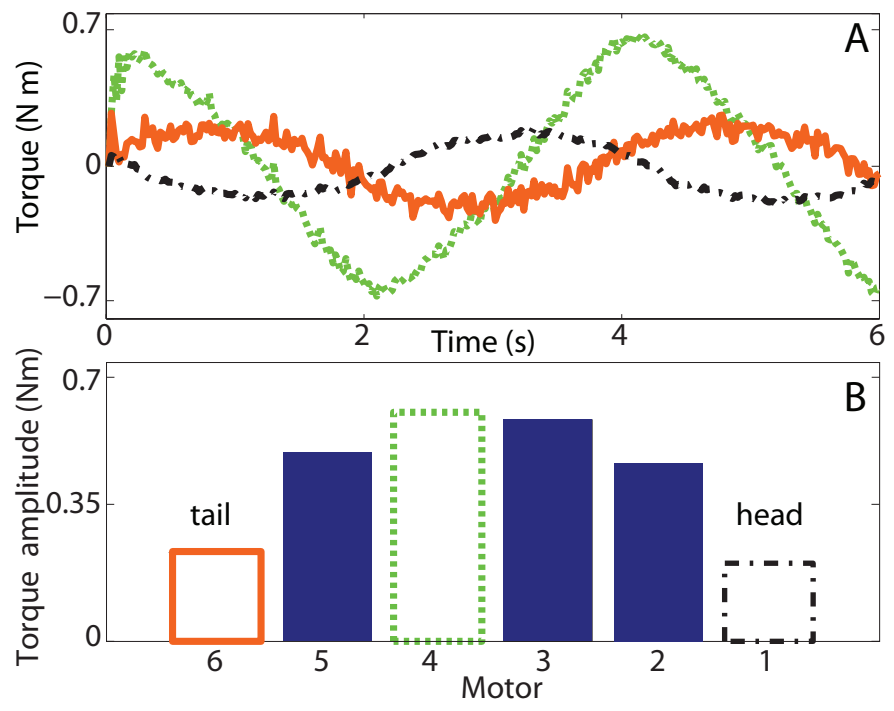

Fig. 9. Motor torque for the simulated 7 segment, 6 motor robot $(f=1 \mathrm{~Hz})$ (A) varying with time. (B) Torque amplitude vs. motor position; orange (solid curve), green (dotted curve), and black (dash-dot curve) correspond to motor 6 (tail), 4 , and 1 (head) with motor position 1 denoting segment number 2 in Fig. 5 and 6.

sandfish uses the same kinematics to move in a variety of media, duplicating the animals control methods and sensing modalities in a robot could lead to more effective locomotion.

The sandfish has a non-trivial shape which suggests changing the morphological characteristics of the robotic device. For example, the cross sectional shape of the sandfish (flat belly and rounded top) have been hypothesized to aid rapid burial into granular media [51]. Our simulated and physical robot can be used to explore the influence of this morphology along with body taper on performance. The robotic simulation can also tune parameters like skin friction and body compliance to identify optimal values which could then be tested with our robot.

\section{CONCLUSION}

Motivated by biological experiments revealing rapid subsurface sand-swimming in the sandfish lizard, we have used numerical simulation as a design tool to build an undulatory sand-swimming device. We used our robot simulation to test whether a device with a finite number of segments (7) could advance using a simple open loop (traveling wave sinusoid) control scheme and calculated the motor torque requirements for the robot. We then built and tested a prototype of the device to validate the biological observations and predictions from the RFT [1] and simulations that limbless body undulations were sufficient to propel the robot forward. Our findings show that the device can swim, and that it translates faster by increasing its oscillation frequency just as the sandfish does. The design tools (numerical model and robot) we developed can generate testable hypotheses of neuromechanical control [52] and improve our understanding of how organisms exploit the solid and fluid-like properties of granular media, enabling the 
construction of robots that can locomote effectively within complex environments.

\section{ACKNOWLEDGMENTS}

We thank Nick Gravish and Chen Li for help with the physics experiments, and we thank Daniel Cohen and Andrew Slatton for help with development of the numerical simulation. We also acknowledge our funding from The Burroughs Wellcome Fund Career Award at the Scientific Interface, NSF Physics of Living Systems grant PHY-0749991, and the Army Research Laboratory (ARL) Micro Autonomous Systems and Technology (MAST) Collaborative Technology Alliance (CTA) under cooperative agreement number W911NF$08-2-0004$.

\section{REFERENCES}

[1] R. Maladen, Y. Ding, C. Li, and D. Goldman, "Undulatory Swimming in Sand: Subsurface Locomotion of the Sandfish Lizard," Science, vol. 325 , no. 5938 , p. $314,2009$.

[2] C. Humphrey and J. Adams, "Robotic Tasks for Chemical, Biological, Radiological, Nuclear and Explosive Incident Response," Advanced Robotics, vol. 23, no. 9, pp. 1217-1232, 2009.

[3] J. McKean, S. Buechel, and L. Gaydos, "Remote sensing and landslide hazard assessment," Photogrammetric engineering and remote sensing, vol. 57, no. 9, pp. 1185-1193, 1991.

[4] G. Metternicht, L. Hurni, and R. Gogu, "Remote sensing of landslides: An analysis of the potential contribution to geo-spatial systems for hazard assessment in mountainous environments," Remote sensing of Environment, vol. 98, no. 2-3, pp. 284-303, 2005 .

[5] A. Ashcheulov, I. Gutsul, and V. Maevski, "Device for monitoring the radiation temperature in coal mines," Journal of Optical Technology, vol. 67 , no. 3 , p. 281,2000

[6] W. Marcus, C. Legleiter, R. Aspinall, J. Boardman, and R. Crabtree, "High spatial resolution hyperspectral mapping of in-stream habitats, depths, and woody debris in mountain streams," Geomorphology, vol. 55, no. 1-4, pp. 363-380, 2003.

[7] R. Ritzmann, R. Quinn, and M. Fischer, "Convergent evolution and locomotion through complex terrain by insects, vertebrates and robots," Arthropod structure and development, vol. 33, no. 3, pp. 361-379, 2004.

[8] R. Siegwart, P. Lamon, T. Estier, M. Lauria, and R. Piguet, "Innovative design for wheeled locomotion in rough terrain," Robotics and Autonomous systems, vol. 40, no. 2-3, pp. 151-162, 2002

[9] J. Wong, "On the study of wheel-soil interaction," Journal of Terramechanics, vol. 21, no. 2, pp. 117-131, 1984.

[10] P. Arena, P. Di Giamberardino, L. Fortuna, F. La Gala, S. Monaco, G. Muscato, A. Rizzo, and R. Ronchini, "Toward a mobile autonomous robotic system for Mars exploration," Planetary and Space Science, vol. 52, no. 1-3, pp. 23-30, 2004.

[11] C. Li, P. B. Umbanhowar, H. Komsuoglu, D. E. Koditschek, and D. I. Goldman, "Sensitive dependence of the motion of a legged robot on granular media," Proceedings of the National Academy of Science, vol. 106, no. 9, pp. 3029-3034, 2009.

[12] C. Li, P. Umbanhowar, H. Komsuoglu, and D. Goldman, "The effect of limb kinematics on the speed of a legged robot on granular media," Experimental Mechanics, pp. 1-11, 2010.

[13] U. Saranli, M. Buehler, and D. Koditschek, "RHex: A simple and highly mobile hexapod robot," The International Journal of Robotics Research, vol. 20 , no. 7 , p. 616,2001

[14] R. Playter, M. Buehler, and M. Raibert, "BigDog," in Unmanned Ground Vehicle Technology VIII, ser. Proceedings of SPIE, D. W. G. Grant R. Gerhart, Charles M. Shoemaker, Ed., vol. 6230, 2006, pp. 62 302O1$62302 \mathrm{O}$.

[15] A. Hoover, E. Steltz, and R. Fearing, "RoACH: An autonomous 2.4 g crawling hexapod robot," in IEEE Intelligent Robots and Systems Conference Proceeding, 2008, pp. 26-33.

[16] A. Saunders, D. I. Goldman, R. J. Full, and M. Buehler, "The rise climbing robot: body and leg design," in Unmanned Systems Technology VIII, G. R. Gerhart, C. M. Shoemaker, and D. W. Gage, Eds., vol. 6230. SPIE, 2006, p. 623017
[17] S. Kim, J. E. Clark, and M. R. Cutkosky, "iSprawl: Design and tuning for high-speed autonomous open-loop running," International Journal of Robotics Research, vol. 25, no. 9, 2006.

[18] S. Hirose and A. Morishima, "Design and control of a mobile robot with an articulated body," The International Journal of Robotics Research, vol. 9, no. 2, p. 99, 1990

[19] Y. Shan and Y. Koren, "Obstacle accommodation motion planning," in Intelligent autonomous systems, IAS-3: proceedings of the international conference, Pittsburgh, Pennsylvania, February 15-18, 1993. Ios Pr Inc, 1993, p. 94

[20] R. Desai, C. Rosenberg, J. Jones, and I. Inc, "Kaa: an autonomous serpentine robot utilizes behavior control," in Proceedings of the 1995 International Conference on Intelligent Robots and Systems, Pittsburgh. Citeseer, 1995.

[21] B. Klaassen and K. Paap, "GMD-SNAKE 2: a snake-like robot driven by wheels and a method for motion control," in Proceedings from the IEEE International Conference On Robotics And Automation, vol. 4, 1999, pp. 3014-3019.

[22] A. Crespi, A. Badertscher, A. Guignard, and A. Ijspeert, "Swimming and crawling with an amphibious snake robot," in IEEE International Conference On Robotics And Automation, vol. 3. Citeseer, 2005, p. 3024.

[23] G. Chirikjian and J. Burdick, "The kinematics of hyper-redundant robot locomotion," IEEE transactions on Robotics and Automation Proceedings, vol. 11, no. 6, pp. 781-793, 1995.

[24] H. Choset, J. Luntz, E. Shammas, T. Rached, D. Hull, and C. Dent, "Design and motion planning for serpentine robots," in Proceedings of SPIE, vol. 3990, 2000, p. 148.

[25] K. Dowling, "Limbless locomotion: learning to crawl," in IEEE International Conference on Robotics and Automation Proceedings, vol. 4, 1999.

[26] H. Kim, D. Shim, and S. Sastry, "Flying robots: modeling, control and decision making," in IEEE International Conference on Robotics and Automation Proceedings., vol. 1, 2002.

[27] J. Zufferey, A. Klaptocz, A. Beyeler, J. Nicoud, and D. Floreano, "A 10-gram vision-based flying robot," Advanced Robotics, vol. 21, no. 14, pp. 1671-1684, 2007.

[28] M. Bekker, Theory of Land Locomotion. The University of Michigan Press, 1956.

[29] D. Tritton, Physical Fluid Dynamics. Oxford University Press, 1989.

[30] D. C. Rapaport, The Art of Molecular Dynamics Simulation, 2nd ed. Cambridge University Press, 2004.

[31] K. Gerritsen, A. van den Bogert, and B. Nigg, "Direct dynamics simulation of the impact phase in heel-toe running," Journal of Biomechanics, vol. 28, no. 6, pp. 661-668, 1995 .

[32] A. Mouazen and M. Nemenyi, "Tillage tool design by the finite element method: Part 1. finite element modelling of soil plastic behaviour," Journal of Agricultural Engineering Research, vol. 72, no. 1, pp. 37-51, 1999.

[33] F. Meysman, J. Middelburg, and C. Heip, "Bioturbation: a fresh look at Darwin's last idea," Trends in Ecology \& Evolution, vol. 21, no. 12, pp. 688-695, 2006.

[34] R. Seymour, P. Withers, and W. Weathers, "Energetics of burrowing, running, and free-living in the Namib Desert golden mole (Eremitalpa namibensis)," Journal of Zoology, vol. 244, no. 01, pp. 107-117, 2001.

[35] K. Dorgan, S. Arwade, and P. Jumars, "Burrowing in marine muds by crack propagation: kinematics and forces," Journal of Experimental Biology, vol. 210, no. 23, p. 4198, 2007.

[36] L. Fielden, "Home range and movement of the Namib Desert golden mole, Eremitalpa granti namibensis (Chrysochloridae)." Journal of Zoology, vol. 223, no. 4, pp. 675-686, 1991.

[37] S. Hawke and R. Farley, "Ecology and behavior of the desert burrowing cockroach, Arenivaga sp. (Dictyoptera, Polyphagidae)," Oecologia, vol. 11 , no. 3, pp. 263-279, 1973 .

[38] E. Arnold, "Identifying the effects of history on adaptation: origins of different sand-diving techniques in lizards," J. Zool., Lond, vol. 235, pp. $351-388,1995$.

[39] C. White, "The influence of foraging mode and arid adaptation on the basal metabolic rates of burrowing mammals," Physiological and Biochemical Zoology, vol. 76, no. 1, pp. 122-134, 2003.

[40] C. Gans, "Tetrapod limblessness: evolution and functional corollaries," Integrative and Comparative Biology, vol. 15, no. 2, p. 455, 1975.

[41] S. Carranza, E. Arnold, P. Geniez, J. Roca, and J. Mateo, "Radiation, multiple dispersal and parallelism in the skinks, Chalcides and Sphenops 
(Squamata: Scincidae), with comments on Scincus and Scincopus and the age of the Sahara Desert," Molecular phylogenetics and evolution, vol. 46, no. 3, pp. 1071-1094, 2008.

[42] J. Gray and G. Hancock, "The propulsion of sea-urchin spermatozoa," Journal of Experimental Biology, vol. 32, no. 4, p. 802, 1955.

[43] N. Cohen and J. Boyle, "Swimming at low Reynolds number: a beginners guide to undulatory locomotion," Contemporary Physics, vol. 51, no. 2, pp. 103-123, 2010.

[44] S. Berri, J. Boyle, M. Tassieri, I. Hope, and N. Cohen, "C. elegans locomotion: a unified multidisciplinary perspective," BMC Neuroscience, vol. 10, no. Suppl 1, p. P16, 2009.

[45] J. Gray and H. Lissmann, "The locomotion of nematodes," Journal of Experimental Biology, vol. 41, no. 1, p. 135, 1964.

[46] B. Jayne, "Kinematics of terrestrial snake locomotion," Copeia, pp. 915927, 1986.

[47] J. Gray, "The mechanism of locomotion in snakes," Journal of Experimental Biology, vol. 23, no. 2, p. 101, 1946.

[48] R. A. Bagnold, The Physics of Blown Sand and Desert Dunes. Methuen and Co. Ltd., 1954.

[49] J. Lee and H. Herrmann, "Angle of repose and angle of marginal stability: molecular dynamics of granular particles," Journal of Physics A: Mathematical and General, vol. 26, pp. 373-383, 1993.

[50] R. Nedderman, Statics and kinematics of granular materials. Cambridge Univ. Pr., 1992.

[51] W. Mosauer, "Adaptive convergence in the sand reptiles of the Sahara and of California: a study in structure and behavior," Copeia, pp. 72-78, 1932.

[52] K. Nishikawa, A. A. Biewener, P. Aerts, A. N. Ahn, H. J. Chiel, M. A. Daley, T. L. Daniel, R. J. Full, M. E. Hale, T. L. Hedrick, A. K. Lappin, T. R. Nichols, R. D. Quinn, R. A. Satterlie, and B. Szymik, "Neuromechanics: an integrative approach for understanding motor control," Integrative and Comparative Biology, vol. 47, no. 1, pp. 16-54, 2007. 